\title{
PREVENCIÓN DEL ABUSO SEXUAL INFANTIL DESDE LA PROMOCIÓN DE LA SALUD SEXUAL
}

\section{Prevention of child sexual abuse as from the promotion of sexual health}

\author{
Anaid Amira Villegas Ramírez* \\ Centro Universitario de los Valles, Universidad de Guadalajara. México \\ Diana Ortiz Sánchez** \\ Centro Universitario de los Valles, Universidad de Guadalajara. México \\ Luz Elena Arreola Miramontes*** \\ Investigadora particular. México
}

\begin{abstract}
Resumen
El presente trabajo informa sobre una intervención con niñas y niños para la enseñanza de estrategias de prevención del Abuso Sexual Infantil (ASI), la promoción de su salud sexual y la capacidad de elaborar prácticas de autocuidado. El trabajo se implementó en una escuela primaria de la región de los Valles en Ameca, Jalisco, participando alumnas y alumnos con edades de 6 a 12 años, con una muestra evaluativa de 24 niños/as, utilizando la herramienta de evaluación Coletas y Verdi de Rodríguez y de la Cruz, 2013. Los resultados confirman la importancia que tiene la prevención del ASI, encontrándose factores protectores que se practican, en contraste con que los niños/as no conocen aspectos esenciales de la sexualidad, el ASI y el cuidado del cuerpo.
\end{abstract}

Palabras clave: Prevención, Abuso Sexual Infantil (ASI), Salud Sexual, Autocuidado.

\begin{abstract}
The present work carries out an intervention with girls and boys for the teaching of strategies for the prevention of Child Sexual Abuse (ASI), the promotion of their sexual health and the ability to develop self-care practices. The work was implemented in a primary school in the Valles region of Ameca, Jalisco, with the participation of female and male students aged 6 to 12, with an evaluative sample of 24 children, using the Coletas and Verdi evaluation tool. de Rodríguez. and de la Cruz, 2013.

The results confirm the importance of the prevention of ASI, even with protective factors that are practiced, but contrasting with the fact that children do not know essential aspects of sexuality, the ASI and caring for the body.
\end{abstract}

Keywords: Prevention, Child Sexual Abuse (ASI), Sexual Health, Self-car

\footnotetext{
* Psicóloga, ORCID: https://orcid.org/0000-0003-2108-9932 anaid.villegas@ valles.udg.mx,

** Psicóloga, ORCID: https://orcid.org/0000-0002-6131-2644 diana.ortiz@ profesores.valles.udg.mx,

*** Egresada de psicología, ORCID: https://orcid.org/0000-0002-3577-6656 arreola1997luci@ gmail.com.
} 


\section{INTRODUCCIÓN}

El abuso sexual infantil (ASI) es una forma de maltrato y se presenta en más ocasiones de las que se llegan a denunciar y procesar teniendo consecuencias a corto plazo en el comportamiento y la conducta del menor, así mismo se estiman consecuencias psicoemocionales perdurables a lo largo de la vida, pudiendo llegar a ser un factor asociado a trastornos de personalidad, depresión, ansiedad, sexuales, adicciones entre otras.

El ASI es una problemática social que tiene alta prevalencia en México, donde este problema social no depende del lugar de residencia, de la cultura, creencias, estatus económico o social sino que es una problemática que no respeta edades, género ni condición socioeconómica y, lamentablemente, los casos a diario siguen creciendo en estándares elevados; es por ello que nos hemos interesado en el trabajo preventivo con los menores de edad, creando una intervención psicoeducativa con el fin de brindar información de la sexualidad con la cual se pretende que los niños conozcan de prácticas protectoras del ASI y que sepan utilizarlas a corto, mediano y largo plazo. Este trabajo tiene la modalidad de taller y cubre temáticas que se han establecido como básicas en otros estudios para la prevención eficaz del abuso sexual infantil, entre dichas temáticas se encuentran: las emociones, las caricias buenas y malas, las partes privadas, los secretos peligrosos, el reconocimiento de nuestra manada (redes de apoyo), que es el Abuso Sexual Infantil (ASI), factores protectores y factores de riesgo. Para el análisis de los resultados, la intervención fue evaluada de manera pretest y post-test, esto para conocer el impacto creado a la población y así mismo corroborar la eficacia de la intervención.

La prevalencia del abuso sexual infantil es una problemática que resulta difícil tener en estimaciones y/o cifras exactas, pues dentro de este tema aparecen una serie de factores que lo impiden, como la falta de denuncias, el encubrimiento por parte de la familia; lo que nos lleva a considerar cifras negras en un alto índice. Aguilar (2009) menciona dos aspectos por los cuales sucede esto: en primer lugar se encuentra la escasa autonomía e imposibilidad de medios tanto del menor como de las personas allegadas y conocedoras del hecho para dar conocimiento de la situación, en segundo lugar se encuentra la elevada cifra de casos sin denunciar. Sin embargo, en un estudio hecho en España, por el centro Reina Sofía para el estudio de violencia, que fue publicado en el año 2004 sobre el análisis estadístico de una muestra de 100 casos de abuso sexual en la infancia, donde los sujetos tenían una edad de entre 8 y 18 años, en los cuales eran tres cuartas partes mujeres (72\%) en cual se encontró que: (1) la edad con más frecuencia de casos va de los 8 a 11 años, (2) los sujetos presentan desarrollo cognitivo normal pero con una frecuencia de fracaso escolar del $18 \%$. Respecto a la familia se encontró que el $27 \%$ contaba con desestructuración y el $57 \%$ con nivel cultural bajo, que conlleva una prevalencia al incesto (Aguilar, 2009). Tras la misma búsqueda de cifras e investigación del fenómeno social se encontró que en un estudio de incidencia del abuso sexual denunciado en mujeres y hombres de los años 1998 al 2003, se descubrió una mayor incidencia hacia las mujeres que a los hombres por el factor asociado del patrón socio-cultural, en donde se considera a la mujer como un objeto sexual, que tomándose en cuenta por parte del contexto social en la cultura mexicana la mujer interactúa con un rol de ser sumisa, por lo cual se encuentra como factor determinante para el sufrimiento de un acto violento que incide principalmente al abuso sexual, mientras que a diferencia si los hombres pasan por un acto violento que conlleve acto sexual, el implicado no denuncia por el estigma puesto por la sociedad y el cuestionamiento de su hombría y condición social.

Tras conocer el impacto social que conlleva tanto para mujeres y hombres un abuso sexual que en este caso es a temprana edad resurge con importancia agregar y destacar que en el año 2016 la Organización para la Cooperación y el Desarrollo Económico (OCDE), reporta que México ocupa el primer lugar a nivel mundial en abuso sexual, violencia física y homicidio de menores de 14 años con 4.5 millones de reportes.

Pasando a las cuestiones legales y de denuncia del ASI, en el Estado de Jalisco, México, se ha encontrado el registro de 221 casos en el mes de marzo de 2019, lo que representa un incremento del $26.3 \%$ con respecto al mes de febrero del mismo año, donde el número de carpetas de investigación por este delito 
fue de 215. Durante el primer trimestre de 2019, se han registrado 611 casos de abuso sexual infantil, de los cuales el $60.2 \%$ se concentran en los municipios del Área Metropolitana de Guadalajara. En promedio han ocurrido 6.7 delitos de este tipo por día, en el primer trimestre del año. Cabe mencionar que, en el Estado, el delito de abuso sexual infantil ha mantenido cifras similares, teniendo que en el 2016 se abrieron 2,345 carpetas de investigación, para el 2017 fueron 2,321 y en el 2018 un total de 2,229 (INEGI, 2019). Frente a lo antepuesto resaltamos la importancia de continuar con el trabajo de intervención sobre la prevención del ASI que se dedique a trabajar con menores de edad mediante un taller con la utilización de material didáctico atractivo para el menor, para que pueda generar, interiorizar y normalizar los términos que incluye su propia sexualidad para que esto sea de impacto en su manera de comunicar o referir lo que amenaza su bienestar de la mano de prácticas de autocuidado personal y sexual que le proponga la disminución de factores de riesgo, de sufrir un abuso sexual infantil.

El abuso sexual infantil es una problemática social que en múltiples estudios ha sido investigada por su extensión en secuelas que se hacen presentes en las víctimas, es un fenómeno social que no solo compete a su estudio al área de psicología sino también a las áreas legales y de salud en su mayor proporción. La Organización Mundial de la Salud (OMS) en el año 2010 trabajó y dedicó su interés para definir el maltrato infantil como: "aquel abuso y desatención de que son objetos los menores de 18 años, incluye todos los tipos de maltrato físico y psicológico, abuso sexual, desatención, negligencia y explotación comercial o dignidad de un niño, o poner en peligro su supervivencia, en el contexto de una relación de responsabilidad, confianza o poder" (OMS, 2010 citado en Martínez-Moya, 2016). Tras este trabajo realizado por la OMS la convención de los derechos de los niños en México define el término de violencia en su artículo número 9 , párrafo 1 , como toda forma de perjuicio o abuso físico o mental, descuido y/ o trato negligente, malos tratos o explotación, e incluso el abuso sexual, en donde genera una definición específica del ASI que se explica como una forma de perjuicio o abuso físico equiparable a un tipo de violencia (Martínez-Moya, 2016). Cuando la OMS dedicó de su tiempo y género una visión tras el trabajo de un fenómeno social que está resurgiendo de manera impactante y amenazaba la integridad de los menores de edad exhortó que los gobiernos deberían implementar programas de educación sexual en donde la información vaya dirigida tanto como para maestros, padres y niños (OMS, 2012). Siguiendo las recomendaciones anteriores, las instituciones educativas en México desde el quinto grado de primaria han implementado clases con la impartición de temas sobre la educación sexual, con la visión de prevenir el embarazo precoz, enfermedades de transmisión sexual y prácticas sexuales de riesgo (Rodríguez y Safora, 2009). Para los investigadores Estes y Weiner (2001), es poco el trabajo que se ha asignado hacia el fenómeno que amenaza la población infantil, ya que hacen mención que el abuso sexual no para ahí sino que otras de las formas de lastimar a los menores de edad son la pornografía y prostitución. Dichos tipos de violencia se asocian de manera directa a lo que es el abuso sexual infantil. En efecto, cuando se habla de los subtipos de violencia que se ven asociados por el abuso se reconoce que son una violación a su máximo nivel de sus derechos humanos. Sin embargo, la verdadera gravedad del abuso sexual está oculta, debido a la naturaleza que le ha dado la sociedad como sensible y prohibida. Los casos que se registran a diario en el país son solo una mínima parte de los ocurren en la sociedad, pero la mayoría de los niños/as y familias que se ven implicadas deciden no denunciar los casos por miedo, estigma y falta de confianza a la autoridad.

Uno de los organismos internacionales que velan por el bienestar de los menores de edad es el Fondo de las Naciones Unidas para la Infancia (UNICEF), que trabaja para prevenir la violencia sexual incorporando a diferentes sectores del gobierno como lo son; la justicia, el bienestar social, la educación y la salud, así como los legisladores, la sociedad civil, los líderes comunitarios, los grupos religiosos, el sector privado, los medios de comunicación, las familias y los propios niños. Así mismo, apoya a los gobiernos en el fortalecimiento de los sistemas de protección de la infancia a nivel nacional y local, incluyendo leyes, políticas, reglamentos y la prestación de servicios integrales a los niños que son víctimas de estos hechos (UNICEF, 2015). 
Tras conocer el impacto provocado por el abuso sexual infantil las asociaciones gubernamentales se han visto más sensibles ante el suceso y han trabajado para establecer una sentencia a toda aquella persona que es considerado/a agresor del delito, esto sin importar parentescos familiares.

Con lo anterior llevado a cabo se trató de impactar en la vida de cada uno de los estudiantes que libremente participaron y que con entusiasmo esperaban cada semana las actividades y trabajos.

\section{MÉTODO}

Primeramente en este proceso fue asignado un tipo de estudio el cual corresponde de corte mixto debido a que una de las intenciones que se plantean en el proyecto es encontrar la cantidad de alumnos que cuentan con información adecuada de la sexualidad para su protección, así mismo se describe un corte cualitativo en el que se rescató información relevante de qué es lo que conocen, cuáles son sus conductas ante la sexualidad y qué prácticas de autocuidado tienen y cuáles no, también en este proceso se describen características que pueden encontrarse de factores de protección y/o factores de riesgo en los participantes. La población con la cual se trabajó se encuentra en los grados de primero a sexto grado de primaria, en un rango de edad de los 6 a los 12 años de edad, niñas y niños, alumnos de la Escuela Primaria Ramón Durán de la localidad de San Antonio Matute, pertenecientes al Municipio de Ameca, Jalisco, México.

Tras identificar una población extensa de cada grupo se determinó que la evaluación se llevara a cabo solo con un grupo muestra el cual fue conformado por dos niñas y dos niños de cada grado escolar, estos fueron elegidos de manera al azar tomando en cuenta de que su asistencia a clases fuera de manera regular para evitar sesgos en la investigación, en donde se obtuvo una muestra de 34 participantes en total. Posterior de asignar el grupo muestra, se comenzó evaluando los conocimientos previos a la aplicación del taller para conocer si el impacto causado al término de la intervención. Para evaluar se utilizó la prueba psicométrica que ha sido estandarizada en población mexicana, que lleva por nombre Verdi y Coletas de Rodríguez y de la Cruz (2013) prueba que ha sido creada con implicaciones al juego, pero permiten evaluar cuantitativamente y así mismo cualitativamente los conocimientos de los niños y niñas. Se ha creado con dicha estrategia con el fin de que los niños y niñas no sientan una indagación profunda al tema de sexualidad en su vida, otro aspecto importante que se trabaja en esta prueba es que no se percibe como examen o evaluación sino como un simple juego. Es una prueba que está compuesta de tres áreas fundamentales para trabajar el abuso sexual infantil, las áreas que se trabajan son: 1. Prevención y Psicoeducación. 2. Detección. 3. Tratamiento mediante el enfoque cognitivo-conductual. Cada una de las pruebas aplicadas requirió de la observación directa, debido a que los juegos/subpruebas son visuales y comportamentales en donde se pidió el consentimiento de los padres de familia para grabar las sesiones y así, posteriormente, hacer el análisis y evaluación de cada una.

\section{CONOCIENDO A LA POBLACIÓN DE TRABAJO}

La participación de la población es indispensable para que la investigación llegue a buen término, en este caso, fue la Escuela Primaria localizada en San Antonio Matute, una población rural del municipio de Ameca en el estado de Jalisco quién decidió, por parte de la dirección de la institución apoyar para que el proyecto se implementará con una muestra representativa de sus alumnos. En un primero momento se tuvo una reunión con la planta docente para informar sobre el proyecto de investigación para posteriormente tener una reunión informativa y de firma de consentimiento informado por parte de los padres de familia, en donde se acordaron fechas y momentos del día para intervenir, buscando el no interferir en las clases mucho tiempo; así se llegó al acuerdo de trabajar los días lunes donde una parte del equipo de trabajo estaría interviniendo con el resto de los estudiantes de cada grupo que no pertenecían al grupo muestra, a la par, los niños del grupo muestra eran asignados a otras labores fuera del aula de clase para que no interfiriera con la investigación. Una vez a la semana, durante 4 semanas, se trabajaba con el grupo muestra, siendo el día 30 de octubre la primera sesión con el grupo muestra, con quienes se presentaron los integrantes del equipo de trabajo, que eran estudiantes de prácticas de psicología educativa 
y psicología social de la licenciatura en Psicología del Centro Universitario de los Valles de la Universidad de Guadalajara, Iniciando el trabajo se aplicó la primera evaluación a niños y niñas (pretest).

\section{DESCRIBIENDO LOS BLOQUES DE TRABAJO}

Los bloques que corresponden a cada sesión se integraron con diferentes actividades lúdicas que permitían a los niños conocer nueva información sobre la sexualidad y no solo eso sino también les permitía internalizar el comportamiento de protección para cuidar de su persona a pesar de su corta edad. En la primera sesión se trabajaron las emociones culminando de manera satisfactoria, en donde ellos tuvieron la oportunidad de hablar sobre las emociones que identificaban, qué situaciones las generan y para que sirve cada una de ellas. Para éste trabajo se crearon marionetas en donde cada niño expuso una emoción que tenía la función de proteger al menor si se identificaba y utilizaba, además les permitía identificar y desarrollar la habilidad para comunicar lo que sienten, para ello se les planteó qué harían en caso de que una persona adulta quisiera hacerles daño, en donde la mayoría respondió "le diría a mis papás que alguien me quiere hacer daño", esto en cierta forma permite pensar en los beneficios que genera la comunicación directa con la familia debido a que puede limitar prácticas de daño personal.

En la segunda sesión se trató el tema de autocuidado, en donde el trabajo consistió en hablarle a los niños de las partes de su cuerpo, el nombre de cada parte privada y no privada, que los identifica como niñas y niños, así como las diferencias, siendo éste un tema que alzó revuelo entre los participantes, sin excepción de edad o grado puesto que los participantes no hacían referencia de las partes que constituye su cuerpo, en específico las partes privadas, por sus nombres sino que les asignaban un sobre nombre o apodo y en ocasiones no los reconocían. Siempre se buscó que al hablar del tema se hiciera de manera respetuosa tanto del grupo de trabajo hacia los participantes como viceversa. En la sesión 3 para trabajar de manera ligada la identificación de las caricia buenas y malas en donde se utilizó material impreso de niñas y niños jugando para que pusieran círculos de colores, rojo para identificar las partes que no se deben tocar y verde para las partes de su cuerpo que no son las privadas y que si ella/él lo considera pueden tocar las demás personas. En este apartado se obtuvo información relevante por su buena aceptación ante conceptos de la sexualidad y se les planteó la idea de que si una persona toca o intenta tocar alguna de las partes rojas a pesar de que sea tu familiar se lo deben contar a alguien.

A los días de esta sesión padres de familia se acercaron al grupo de trabajo para dar gracias por enseñarle a sus hijos acerca de las partes del cuerpo ya que ellos fueron y contaron en casa lo que habían aprendido. Estas acciones, de manera personal, nos permiten identificar que el trabajo va por buen camino y que se debe seguir perseverando para impactar en el bienestar psicológico, social y físico del menor.

En la cuarta sesión se trató el tema de sexualidad, iniciando con la identificación de los conocimientos que ellos tenían sobre este concepto, como se pueden cuidar, las ocasiones donde la sexualidad se ve afectada (pequeño acercamiento al término ASI) y qué prácticas les permite estar alejados de sufrir una afectación (factores de protección). Tras el buen desarrollo que se implementó en la sesión, los niños hablaban de la importancia de no callar lo que les pasa; pasando a tratar el tema de los secretos/pactos buenos y malos y la importancia de hablarlo con una persona de confianza. Conforme pasaron las sesiones nos permitió observar la normalización que los niños tenían de los términos, su participación cada vez más activa.

\section{RESULTADOS Y DISCUSIÓN}

Al culminar con los cuatro bloques el grupo manifestó disposición de conocer y hacer parte de ellos la información que cambió su perspectiva, se considera que a pesar de su corta edad, reconocen la importancia de la sexualidad en sus vidas, reconociendo su propio cuerpo ya que ellos necesitan explicar lo que en distintas ocasiones experimentan (sensaciones, emociones, sentimientos, conductas). Ante este aspecto se ha aprovechado dicho interés para ejercer información de calidad que les dé a los niños pautas de cómo actuar en situaciones riesgo que en este caso acontece a un tipo de violencia como lo es el Abuso Sexual. En este apartado como ya se ha mencionado 
anteriormente los alcances de la investigación se tornan hacia dos enfoques de evaluación de la información primeramente se encuentra el cuantitativo y posterior el cualitativo. El primero se ha encargado de resolver y asignar una frecuencia a una situación, es por tanto que se considera necesario integrar las siguientes gráficas que llevarán su interpretación y generarán respuesta a las preguntas establecidas.

En la pregunta general que se ha planteado ¿Cuáles son los conocimientos que tienen los niños y niñas de su sexualidad que les permiten cuidar de sí mismos?, tras las pruebas aplicadas se da la oportunidad de comenzar a dar respuesta a esta pregunta, en la figura 1.
Se encontró que del total de los 24 niños y niñas evaluados 19 de ellos (79.2\%) puede identificar cada parte de su cuerpo y sus diferencias con el sexo opuesto, por otra parte, 4 de ellos (16.7\%) reconoce casi todas las partes de su cuerpo y solo 1 de ellos (4.2\%) logra conocer las partes básicas del cuerpo; Deza-Villanueva (2005) menciona que para fortalecer al niño ante la prevención del abuso sexual infantil es necesario que conozca: sobre el reconocimiento de las partes de su cuerpo, logre identificar las partes íntimas (genitales) y ante todo que sepa reconocer los tipos de caricias que se dan en el cuerpo (partes privadas o visibles) y qué es la emoción que esto provoca.

\section{Figura 1}

Porcentaje de niños que logran identificar las partes de su cuerpo, considerado como factor protector.

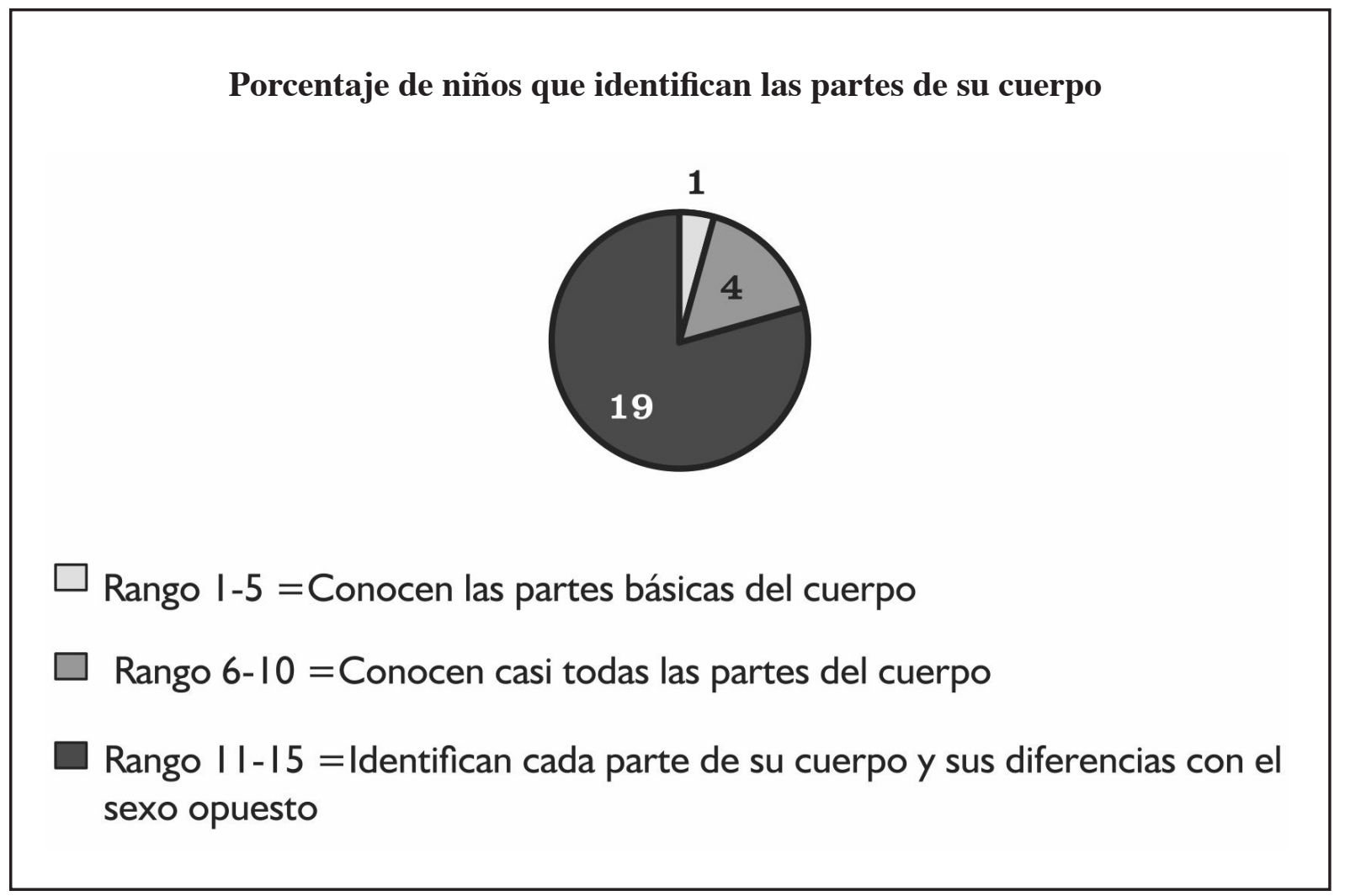




\section{Figura 2}

Cantidad de niños que identifican el sistema sexual

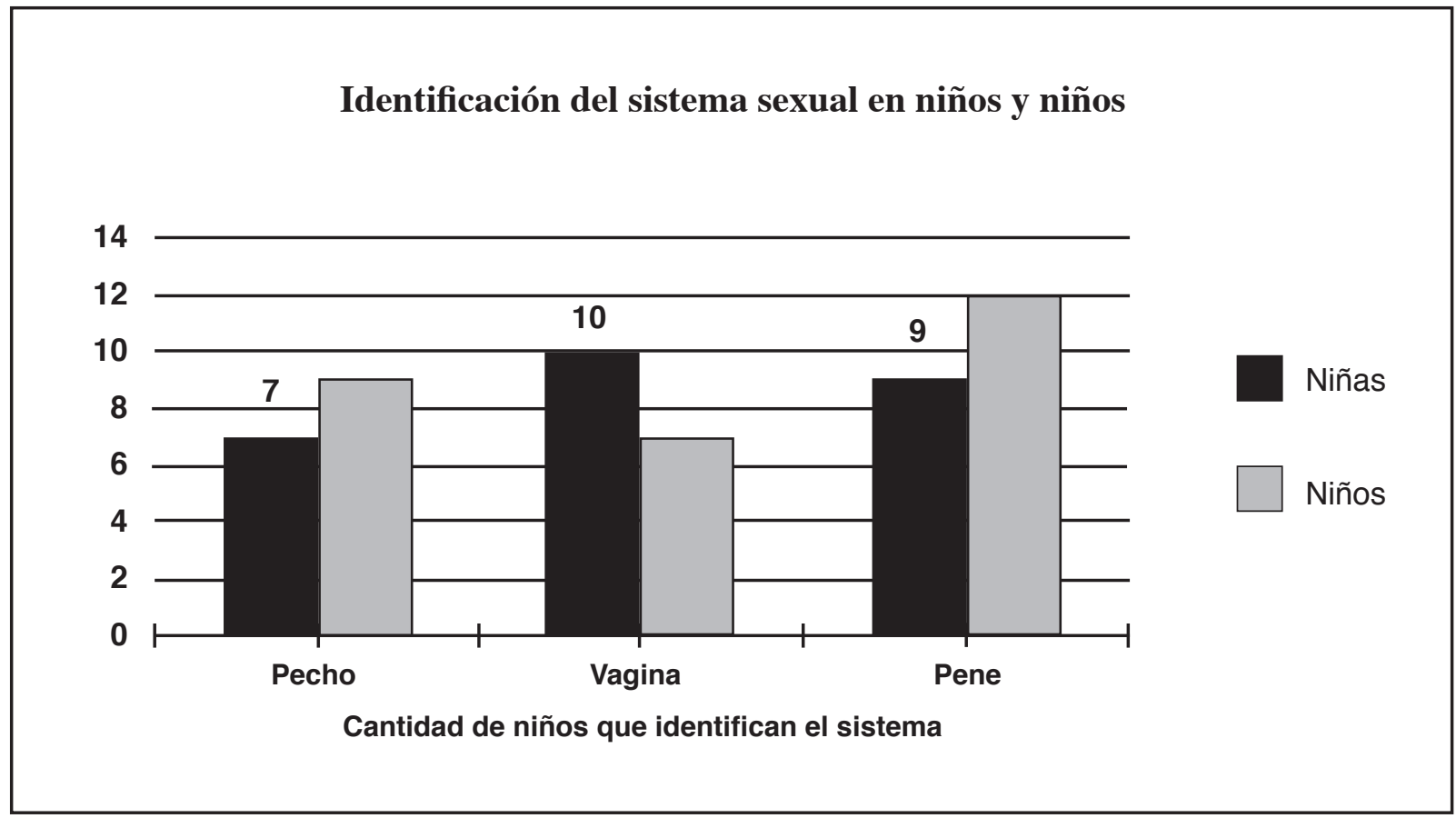

Entonces contextualizando la información de estas gráficas a la respuesta general se puede considerar que la información con la que los niños cuentan en un rango de edad de los 6 a los 12 años es: los nombres de sus partes íntimas, las diferencias que tienen entre ellos y quienes debe tocarlos y con qué personas deben admitir tocamientos. Realmente, aunque suena basta esta información es muy poca para la edad en la que los niños se encuentran para protegerse, sin embargo, los padres de familia y maestros quienes son las personas más cercanas y encargadas de la educación de los menores no prestan interés e importancia de educar para la sexualidad debido a que siguen viendo con error lo que es este tema y así mismo lo convierten en factor de riesgo para el menor de edad. Entrando al contexto de dar respuesta a preguntas más específicas de la problemática, se dará inicio con la siguiente; ¿Cantidad de alumnos que cuenta con información de la sexualidad confiable? Dicha información se ha rescatado de la suma de los puntajes de todas las subpruebas la cual refleja la cantidad de alumnos que conocen de sexualidad básica como lo son partes del cuerpo, caricias, emociones, secretos, protección y riesgos. Los datos confirman en este caso que solo de los 24 alumnos que han sido evaluado 16 conocen de lo fundamental que demanda el tema de sexualidad, con lo cual los 8 alumnos restantes se encuentran en predisposición de factor de riesgo a un abuso (Figura $3)$. 


\section{Figura 3}

Cantidad de alumnos que cuentan con información confiable de la sexualidad

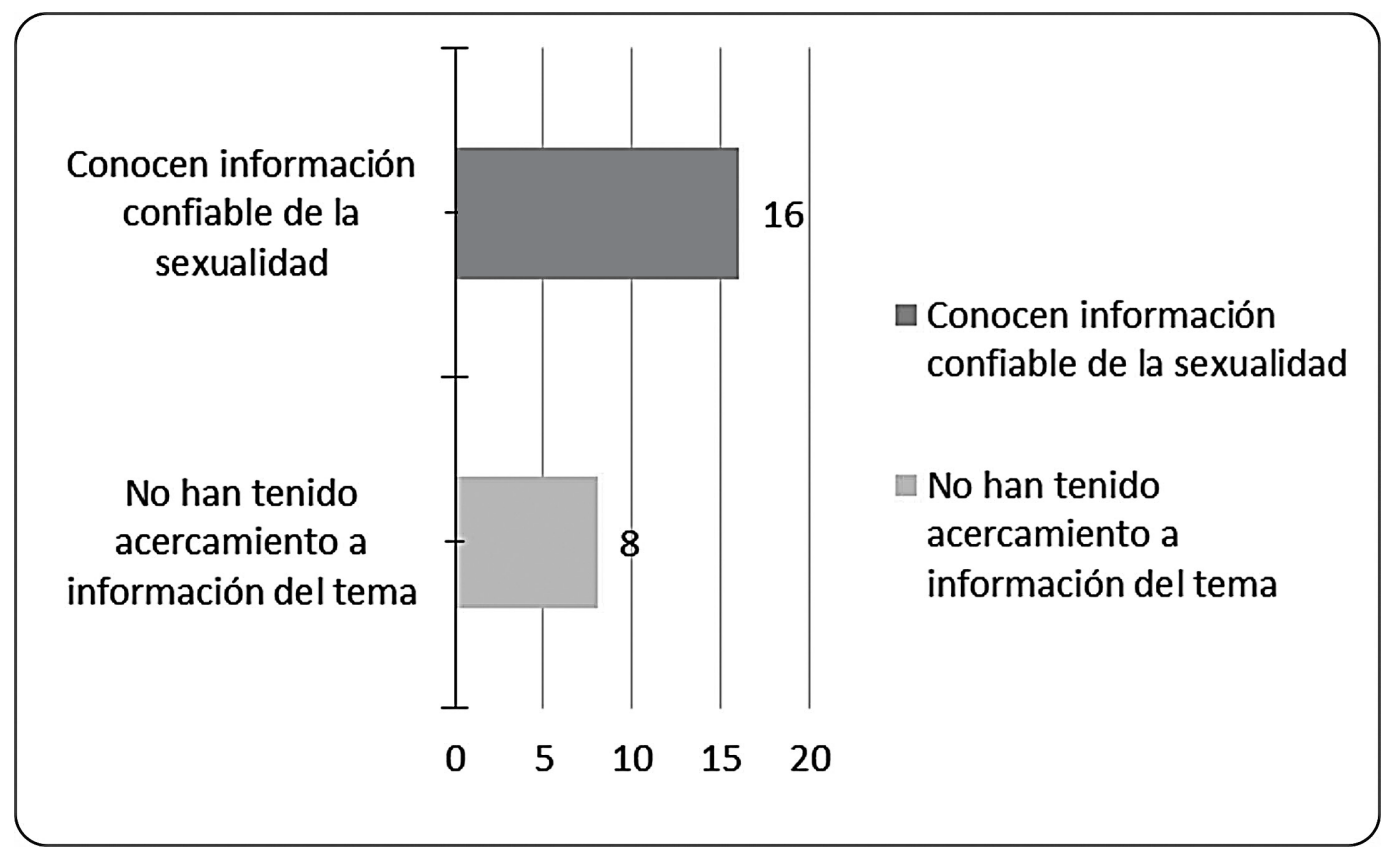

Además, ante este misma gráfica se logró mediante las observaciones y anotaciones rescatar que la persona que se encarga de educar en la sexualidad es la Madre de familia, esto también asignado por un patrón social que impone a la mujer como la única persona encargada de la educación de los hijos, es aquí también donde se considera la importancia de las representaciones sociales donde se establece que si el hijo es abusado es porque la madre lo descuido o no lo supo educar de la mejor manera estableciendo una etiqueta de mala madre. Dando paso a la explicación de las subpruebas que conllevan metodología mixta se ha creado en conjunto de las siguientes gráficas una red temática la cual se irá explicando conforme se vayan contestando las siguientes preguntas que conllevan dicha metodología. La siguiente pregunta por contestar es ¿Qué emociones les permiten a los niños afrontar situaciones que los ponen en riesgo o así mismo le ayudan a evitar un ASI?

Durante la intervención se ha encontrado (Figura 4) que los niños en situaciones de peligro en este caso manejadas por la prueba como caricias malas tienen una capacidad de referir su sentir mediante la emoción de ira, reconociéndola como emoción protectora ya que genera comportamientos inmediatos que permiten observar situaciones de peligro ante terceros. En observaciones realizadas, los menores verbalizaron la siguiente frase: "si una persona me llega a tocar las partes de mi cuerpo, que son solo mías me dará mucho coraje pero le diré a mi papá para que se arregle con él"; a pesar de que no se considera correctamente los golpes para solucionar problemáticas, ante esta frase se puede interpretar: "tus actos me harán daño, y aunque me enojaré mucho, tengo personas quienes van a cobrar los hechos realizados", lo cual involucra expresar el acto (comunicación asertiva). Tras esta interpretación, se puede considerar que es más benéfico que los menores utilicen la irá a comparación de la emoción tristeza, ya que ésta es considerada como inhibidora y solo permite guardar silencio en caso de presencia de un acto sexual. A pesar de que se encuentra como segunda emoción determinante de riego ante un abuso, es un menor porcentaje de niños con el cual se trabajará para cambiar la perspectiva de la expresión de emociones ante situaciones que atentan hacia su bienestar. 


\section{Figura 4}

Emociones consideradas como protectoras para la Prevención del ASI

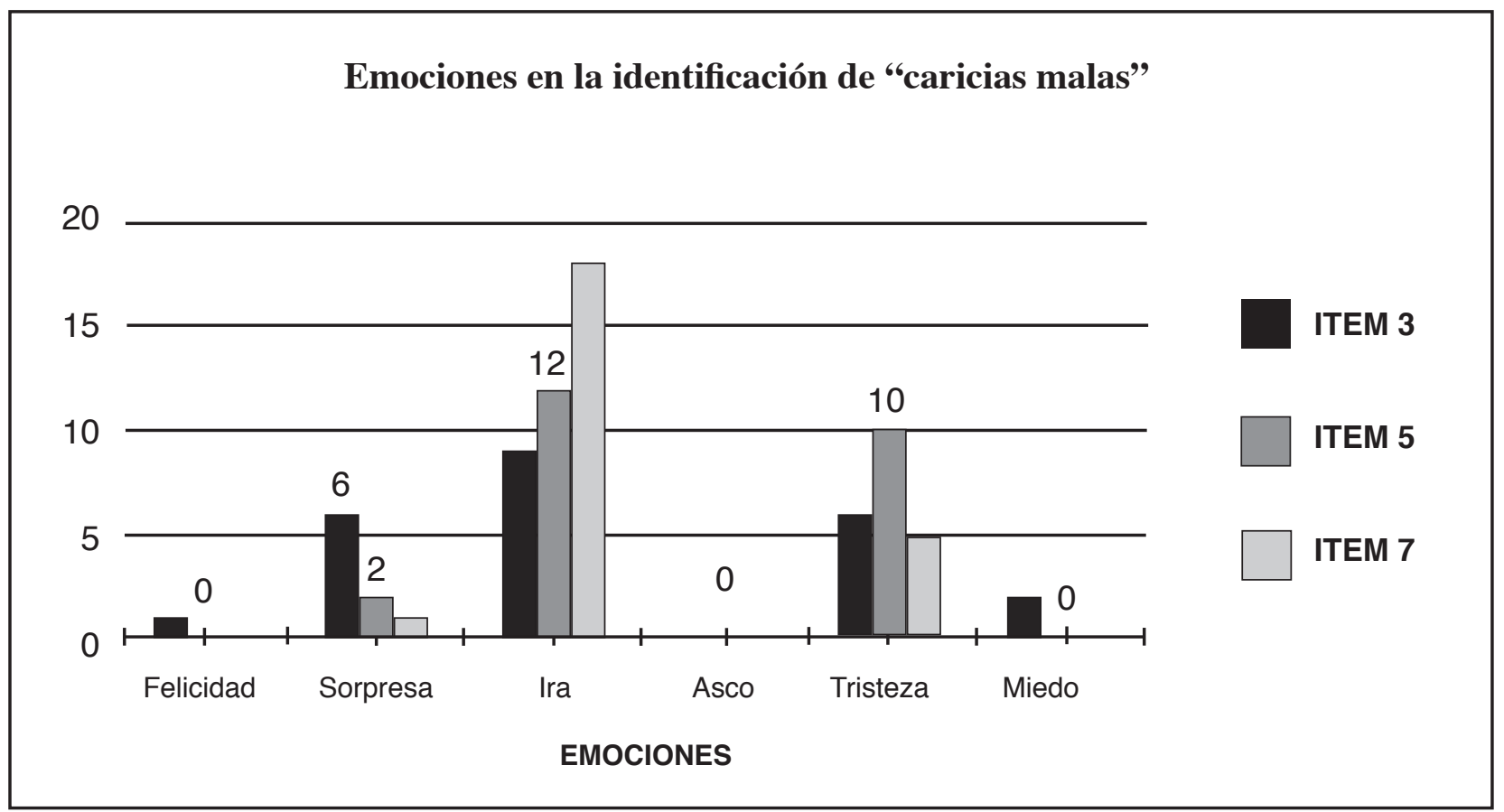

La siguiente pregunta fue si los niños y niñas pueden identificar las caricias malas y buenas o no pueden identificarlas; dentro de la primera aplicación y como lo muestra la figura 5, se encuentra en la población evaluada, 23 de ellos (95.8\%) logran identificar las partes de su cuerpo que no deben de ser tocadas por otras personas, no así 1 de ellos (4.2\%) quien no identificó o diferenció las caricias buenas de las caricias malas. Posterior a esta actividad se les hizo mención que todo su cuerpo debe ser cuidado y que independientemente de quién o quiénes intenten tocar su cuerpo ellos podían decidir a acceder o no a ser tocados, haciendo siempre la diferencia entre lo que es una caricia buena, que es una caricia que te hace sentir bien anímica y físicamente bien, de una caricia mala, la cual siempre le iba hacer sentir física y anímicamente mal. 


\section{Figura 5}

Porcentaje de niños que identificas las caricias buenas y las caricias malas

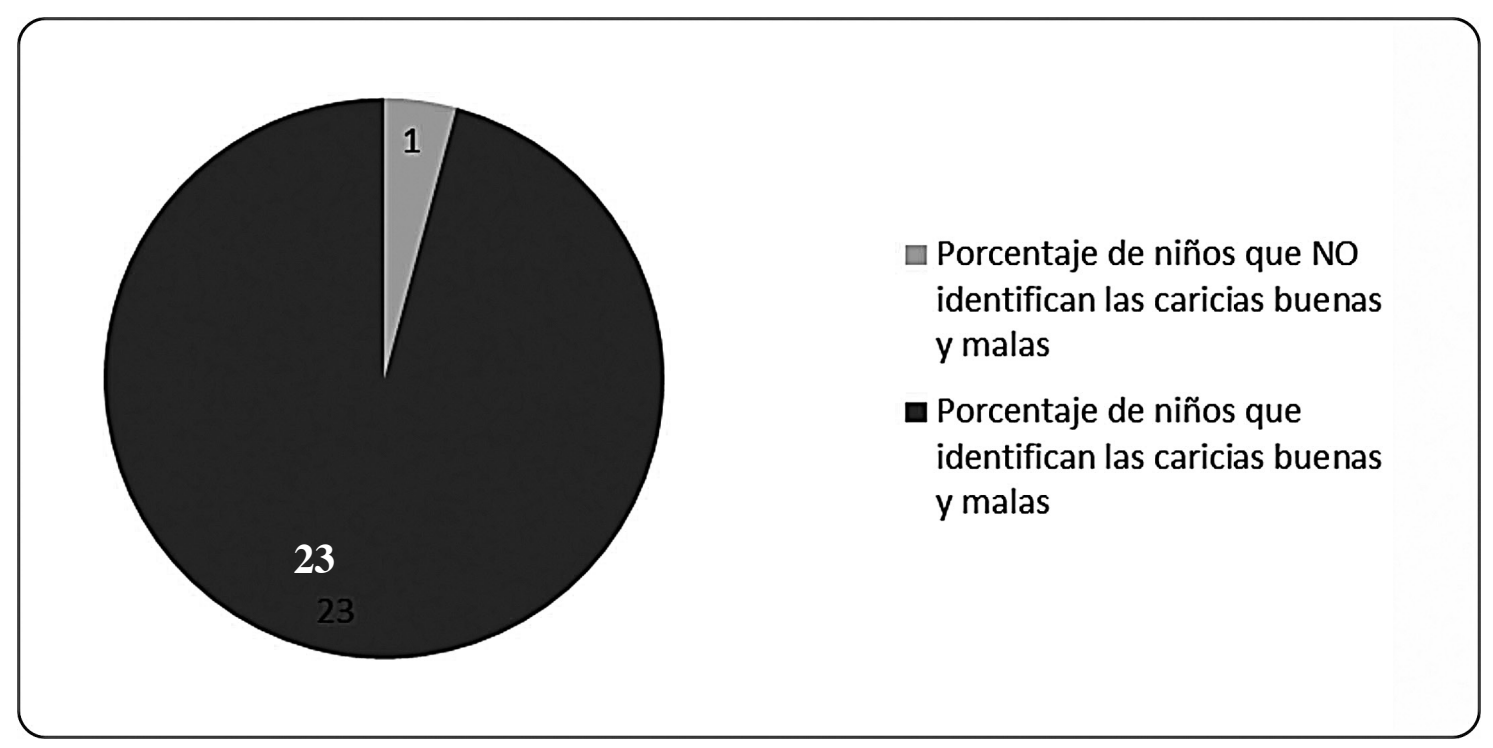

En base a la estructura del proyecto de prevención de la problemática social, se reconoció la importancia que se asigna a la identificación de las formas de querer y en base qué comportamiento o señales las identifican. Esta subprueba fue una de las evaluadas cualitativamente, la cual refirió los siguientes códigos:

- Personas que están relacionadas amorosamente, ya que se están tocando partes de su cuerpo.

- Relación de esposos es por ser adultos mayores.

- Relación madre e hijo o abuela-nieto, por diferencia de edades.

Estos códigos permiten reconocer que los niños y niñas, atribuyen que una persona consiente que otra le toque partes de su cuerpo por la razón de estar enamorados, este código en cierto grado preocupa y es necesario trabajar con exactitud dado que se expone como factor de riesgo ya que los niños en este caso pueden confundir que tocar es demostrar cariño y se conoce en estudios que los abusadores en su mayoría se aprovechan de la incapacidad de razonamiento de los niños para interpretar los tocamientos como referencia de una forma de querer. Dando respuesta a la última pregunta de investigación, ¿Qué prácticas de cuidado personal los niños tienen para prevenirse de un abuso sexual por otra persona?, comentaremos que la respuesta de esta pregunta ha sido analizada en las observaciones directas, reconociéndose lo siguiente:

- No compartir mucho tiempo con una sola persona.

- Quién debe tocar sus partes privadas y quiénes no.

- Identifican quiénes son las personas a las cuales pueden hablarles con confianza en caso de sospechar de un factor de riesgo del ASI.

Posterior de reconocer toda la información plasmada anteriormente se logró crear una red temática (Figura 1) con la información recabada en la prueba, las notas de observaciones y el conjunto del material bibliográfico consultado, en donde resulta importante mencionar que el mayor porcentaje de los niños evaluados presentan conocimientos e información que les permite crear prácticas de protección del ASI. 


\section{Figura 6}

Red temática sobre los conocimientos que tienen las niñas y los niños sobre la sexualidad que les permiten generar prácticas de auto cuidado



\section{CONCLUSIONES}

Al término del trabajo concluimos que cuanta más información tienen los niños sobre la sexualidad más prácticas de autocuidado presentan, lo cual indica que el niño está menos propenso a sufrir de un abuso, así que entre mayor claridad de información tengan los menores en caso de presentar o sospechar de un abuso no se detendrán a pedir ayuda, ellos lograran identificar el riesgo presentado y avisan de inmediato a su manada (red de apoyo). Así mismo, nos percatamos que a pesar de que tienen poca información sobre la sexualidad, ésta es confiable, lo que significa que es básica dicha información. Ello nos permite apelar a que este y otro tipo de trabajos sobre el tema se siga implementando en las aulas de la educación básica, pues con excepción de nuestro trabajo, no se aborda la prevención del ASI en las escuelas primarias de la región de los Valles. Con pleno conocimiento de que este es un trabajo arduo que está iniciando y que se cuenta con el apoyo e interés de los padres de familia y de las escuelas primarias, también apelamos a la participación de los distintos niveles de gobierno, las autoridades educativas en conjunto con los padres de familia, a manera de acciones que se pongan en práctica y se tome cartas en el asunto y se deje de simulaciones, que haga valer los derechos de los niños, porque para ser un país diferente se necesita educar niños diferentes, niños libres de amenazas, preocupándose solo por aprender, estudiar y disfrutar cada etapa de la vida. El trabajo que falta es mucho y debe ser de manera integral donde el niño se capacite sobre el ASI y su prevención pero también el trabajo debe ser en casa y en las escuelas, capacitando a los padres y a los profesores ya que ellos son los principales personas 
que educan e informan a los niños y son ellos los que con su respaldo, fomento y normalización de la educación sexual, desde edades tempranas, aportarían las bases de una educación sexual para la prevención del ASI.

\section{REFERENCIAS}

Aguilar, M. (2009). Abuso Sexual en la Infancia. Revista Anales del Derecho, 27 (1), pp. 221Universidad de Murcia.

Deza-Villanueva, S. (2005). Factores protectores en la prevención del abuso sexual infantil. Liberabit, 11(11), 19-24.

Estes, R., \& Weiner, N. (2001). ThE commercial sexual Exploitation of Children in the USA, Canada and México. National study.

Instituto de Información Estadística y Geográfica de Jalisco. (2019). Delitos de Abuso Sexual Infantil en Jalisco. México. Gobierno del Estado.
Martínez-Moya, L. (2016). El abuso sexual infantil en México: Limitaciones de la intervención estatal. Instituto de investigaciones Jurídicas.

Organización Mundial de la Salud. (2012). Prevenir el embarazo precoz y los resultados reproductivos adversos en adolescentes en los países en desarrollo: las evidencias. OMS.

Organización para la Cooperación y Desarrollo Económico. (2014). México primer lugar a nivel mundial en abuso sexual a menores: OCDE. Regeneración.

Organización para la Cooperación y Desarrollo Económico. (2016). México primer lugar a nivel mundial en abuso sexual a menores: OCDE. Regeneración.

Rodríguez, A., \& Safora, O. (2009). Embarazo en la adolescencia: dos caras de una moneda. Científico Técnica.

UNICEF. (2015). Protección infantil contra la violencia, la explotación y el abuso. Unicef.

Fecha de recepción: 25 de octubre 2020

Fecha de aceptación: 10 de noviembre 2020 